\title{
Stability of Negative Solitary Waves for a Generalized Camassa-Holm Equation with Quartic Nonlinearity
}

\author{
Shan Zheng ${ }^{1}$ and Zhengyong Ouyang ${ }^{2}$ \\ ${ }^{1}$ Department of Basic Courses, Guangzhou Maritime Institute, Guangdong 510725, China \\ ${ }^{2}$ Department of Mathematics, Foshan University, Guangdong 528000, China
}

Correspondence should be addressed to Shan Zheng; szheng_math@163.com

Received 6 October 2015; Revised 1 December 2015; Accepted 8 December 2015

Academic Editor: Christian Engstrom

Copyright ( $\odot 2015$ S. Zheng and Z. Ouyang. This is an open access article distributed under the Creative Commons Attribution License, which permits unrestricted use, distribution, and reproduction in any medium, provided the original work is properly cited.

We consider the stability of negative solitary waves to a generalized Camassa-Holm equation with quartic nonlinearity. We obtain the existence of negative solitary waves for any wave speed $c>0$ and some of their qualitative properties and then prove that they are orbitally stable by using a method proposed by Grillakis et al.

\section{Introduction}

The Camassa-Holm equation ( $\mathrm{CH}$ for short)

$$
u_{t}-u_{x x t}+3 u u_{x}=2 u_{x} u_{x x}+u u_{x x x}
$$

was first derived by the method of recursion operators by Fokas and Fuchssteiner for studying completely integrable generalization of the $\mathrm{KdV}$ equation with bi-Hamiltonian structure in [1] and later proposed physically by Camassa and Holm as a model for unidirectional propagation of shallow water waves over a flat bottom in [2], and it was shown that the $\mathrm{CH}$ equation is completely integrable and possesses an infinite number of conservation laws. It is very different from the $\mathrm{KdV}$ equation that the $\mathrm{CH}$ equation has peakon solution and breaking waves (see [2-4]). As mentioned in [5], it is interesting to find that both phenomena of soliton interaction and wave breaking can be exhibited in one mathematical model of shallow water waves. The $\mathrm{CH}$ equation was well studied in view of mathematical point and a lot of results were established. For example, the Cauchy problem for $\mathrm{CH}$ and periodic $\mathrm{CH}$ equation were studied in [6-8], the global weak solutions and global conservative and dissipative solutions were obtained in [9-13], and the peakon and smooth solitary wave solutions were proved to be orbitally stable and interact like solitons [14-17] on the wave breaking; we refer to [3, 1821].

Furthermore, considerable researches have studied the following generalized $\mathrm{CH}$ equation [22-27]:

$$
u_{t}-u_{x x t}+a u^{n} u_{x}=2 u_{x} u_{x x}+u u_{x x x}
$$

they have focused on the stronger nonlinear convection; that is, the nonlinear convection term $u u_{x}$ in (1) has been changed to $u^{n} u_{x}$ in (2), which makes the structure of their solutions change significantly. There are many new nonlinear phenomena arising from (2), such as compacton solitons with compact support, solitons with cusps, or peakons (cf. [28-36]). Four simple ansätze were proposed to obtain abundant solutions: compactons, solitary patterns solutions having infinite slopes or cusps, and solitary waves in [28]. By using bifurcation method, peakons and periodic cusp waves were studied in [32-34]; the explicit expressions of peakons for (2) are given in some special cases. In [35] some new exact peaked solitary waves were derived. By employing polynomial ansätze the periodic wave and peaked solitary waves of (2) were investigated in [36]. The Cauchy problem of (2) was studied in $[37,38]$ and the local existence was established. For $a=3$ and $n=2$, the negative solitary wave to (2) was obtained and proved to be orbitally stable for any 
speed in [22]. Moreover, the stability problem of solitary wave to (2) was investigated as $n=3$ and $a>0$ in [39].

Our study is closely related to the results in [39]. For convenience, we write (2) when $n=3$ in the following form:

$$
u_{t}-u_{x x t}+a u^{3} u_{x}=2 u_{x} u_{x x}+u u_{x x x} .
$$

In [39], when the parameter $a>0$, (3) was shown to be Painlevé nonintegrable and to have positive solitary waves as wave speed $c>\sqrt{10 / a}$. The solitary waves were proved to be unstable when the wave speed $c$ tends to the critical value $\sqrt{10 / a}$ and stable while the wave speed is a little bigger than the critical value.

However, when $a<0$ in (3), the stability problem of the solitary waves has not been solved yet. In this paper, we consider this stability problem. Unlike the results for positive solitary waves in [39], we show that there exist negative solitary waves for any wave speed $c>0$. The point lying in our results is that we can actually determine that the scalar function $d(c)$ (see below) is convex with respect to wave speed $c$; that is, all the negative solitary waves are orbitally stable.

\section{Preliminaries}

2.1. Hamiltonian System and Conservation Laws. Equation (3) can be rewritten in the following Hamiltonian form:

$$
u_{t}=J F^{\prime}(u)
$$

where $J=-\partial_{x}\left(1-\partial_{x}^{2}\right)^{-1}$ is a Hamiltonian operator, $F^{\prime}(u)$ and $E^{\prime}(u)$ (see below) denote Riesz representations of Fréchet derivatives of $F(u)$ and $E(u)$, and

$$
F(u)=\frac{1}{2} \int_{R}\left(\frac{a u^{5}}{10}+u u_{x}^{2}\right) \mathrm{d} x
$$

is a functional of $u$.

Another functional of $u$ is given by

$$
E(u)=\frac{1}{2} \int_{R}\left(u^{2}+u_{x}^{2}\right) \mathrm{d} x,
$$

which can be treated as the kinetic energy of the waves. Both quantities $E(u)$ and $F(u)$ are critically important to the proof of solitary waves, which are shown to be conserved by the following lemma.

Lemma 1. The functionals $E(u)$ and $F(u)$ defined above are conserved quantities under (3).

Proof. Multiplying (3) by $u$ and integrating over $R$ we have

$$
\frac{\mathrm{d}}{\mathrm{d} t}\left(\frac{1}{2} \int_{R}\left(u^{2}+u_{x}^{2}\right) \mathrm{d} x\right)=\frac{\mathrm{d}}{\mathrm{d} t} E(u)=0 .
$$

To show that $F(u)$ is invariant with respect to $t$, we need to use the Hamiltonian structure of (3). It follows from (4) that

$$
\begin{aligned}
\frac{\mathrm{d}}{\mathrm{d} t} F(u(t)) & =\left(F^{\prime}(u), u_{t}\right)=\left(F^{\prime}(u), J F^{\prime}(u)\right) \\
& =-\left(J F^{\prime}(u), F^{\prime}(u)\right) .
\end{aligned}
$$

Using the skew symmetry of Hamiltonian operator $J$ we obtain that

$$
\frac{\mathrm{d}}{\mathrm{d} t} F(u(t))=0
$$

2.2. Definition of Orbital Stability. As already observed by Benjamin and coworkers $[40,41]$, a solitary wave cannot be stable in the strictest sense of the word. To understand this, consider two solitary waves with different height, centered initially at the same point. Since the two waves have different amplitude and they have different velocity, as time passes the two waves will apart, no matter how small the initial difference was. However, in the situation just described, it is evident that two solitary waves with slightly differing height will stay similar in shape during the time evolution. An acceptable notion of stability is given by measuring the difference in shape. This sense of orbital stability was introduced by Benjamin [40]. We say a solitary wave is orbitally stable if a solution $u$ of (3) that is initially sufficiently close to a solitary wave will always stay close to a translation of the solitary wave during the time of evolution. A more mathematically precise definition is given as follows.

Definition 2. The solitary wave $\varphi$ is stable if, for every $\epsilon>0$, there exists a $\delta>0$, such that if $\|u(0)-\varphi\|_{H^{2}} \leq \delta$ and $u \in$ $C\left([0, T) ; H^{2}\right)$ is a solution of $(3)$ for some $T \in(0,+\infty]$ with the initial value $u(0)$, then for all $t \in[0, T)$ one has

$$
\inf _{s}\left\|u-\tau_{s} \varphi\right\|_{H^{2}}<\epsilon
$$

where $\tau_{s} f(x)=f(x-s)$ is a translation of $f$. The solitary $\varphi$ is unstable if $\varphi$ is not stable.

\section{Negative Solitary Waves and Well-Posedness}

3.1. Negative Solitary Waves and Their Properties. Letting $u(x, t)=\varphi(\xi), \xi=x-c t$, and substituting it into (3), it follows that

$$
-c \varphi^{\prime}+c \varphi^{\prime \prime \prime}+a \varphi^{3} \varphi^{\prime}=2 \varphi^{\prime} \varphi^{\prime \prime}+\varphi \varphi^{\prime \prime \prime}
$$

the prime denotes derivative with respect to $\xi$. Integrating the above equation once we have

$$
c \varphi-c \varphi^{\prime \prime}-\frac{a}{4} \varphi^{4}+\varphi \varphi^{\prime \prime}+\frac{\left(\varphi^{\prime}\right)^{2}}{2}=0,
$$

where the integral constant takes zero due to the solitary waves vanish at infinity.

Lemma 3. When the parameter $a<0$ in (3), for any wave speed $c>0$, there exist negative solitary waves $\varphi(x-c t)$ for $(3)$. 


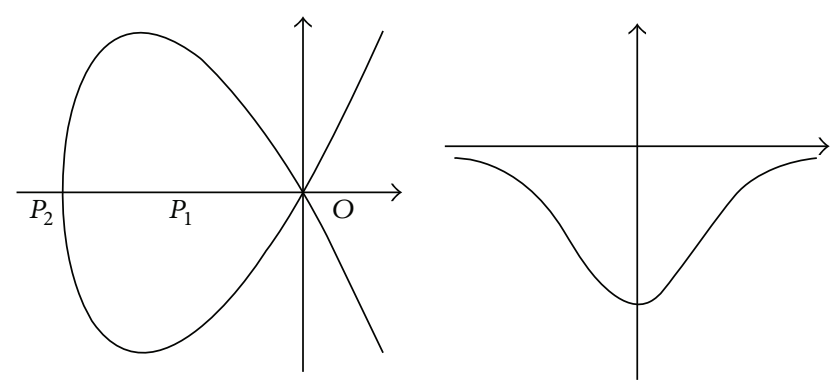

FIgURE 1: Homoclinic orbit and negative solitary wave.

Proof. In fact, we only need to show the existence of homoclinic orbit corresponding to the negative solitary wave. Equation (12) can be rewritten as the following planar system:

$$
\begin{aligned}
& \frac{\mathrm{d} \varphi}{\mathrm{d} \xi}=y, \\
& \frac{\mathrm{d} y}{\mathrm{~d} \xi}=\frac{-c \varphi+(a / 4) \varphi^{4}-y^{2} / 2}{\varphi-c} .
\end{aligned}
$$

By using the transform $\mathrm{d} \xi=(\varphi-c) \mathrm{d} \tau$, system (13) can be rewritten as

$$
\begin{aligned}
& \frac{\mathrm{d} \varphi}{\mathrm{d} \tau}=(\varphi-c) y, \\
& \frac{\mathrm{d} y}{\mathrm{~d} \tau}=-c \varphi+\frac{a}{4} \varphi^{4}-\frac{y^{2}}{2},
\end{aligned}
$$

which is equivalent to system (13) except the singular line $\varphi=c$; it is more convenient to study (14) than (13). The first integral of (14) is

$$
H(\varphi, y)=\frac{a}{10} \varphi^{5}-c \varphi^{2}+(c-\varphi) y^{2}=2 h .
$$

System (13) has two equilibrium points: one at the origin $O(0,0)$ and another given by $P_{1}(\sqrt[3]{4 c / a}, 0)$. By the same bifurcation method used in [32-34], it is easy to determine that $O(0,0)$ is a saddle point, $P_{1}(\sqrt[3]{4 c / a}, 0)$ is a center point, and there is a homoclinic orbit which corresponds to the negative solitary wave (see Figure 1). The homoclinic orbit proceeds from the saddle point $O$, encircles the center $P_{1}$, and returns to the origin. It passes through the point $P_{2}(\sqrt[3]{10 c / a}, 0)$ on $\varphi$-axis.

In spite of the fact that we are not able to obtain explicit expression of solitary wave solution of (3), we can deduce some features of its solitary traveling wave solution for $c>0$ along the lines of ideas in [15], for describing the wave profile qualitatively. Let $\varphi$ be a solitary traveling wave solution of (3). We claim that $\varphi$ has a single minimum; here the minimum of $\varphi$ is used because the solitary wave is negative. For this purpose, based on (15) and the homoclinic orbit starting from the origin, we know that the corresponding negative solitary wave satisfies

$$
\frac{a}{10} \varphi^{5}-c \varphi^{2}+(c-\varphi) y^{2}=0
$$

Since $y=\mathrm{d} \varphi /(\varphi-c) \mathrm{d} \tau=\mathrm{d} \varphi / \mathrm{d} \xi$, it follows from (16) that

$$
\varphi_{\xi}^{2}=\varphi^{2} \frac{c-a \varphi^{3} / 10}{c-\varphi} .
$$

It is easy to determine that $c-a \varphi^{3} / 10$ in (17) has only one real root $-\sqrt[3]{10 c /(-a)}$ which is negative due to the parameter $a<0$ and wave speed $c>0$. It follows that $\varphi_{\xi}$ vanishes precisely at this negative real root, so $\varphi$ has a unique minimum wave lowness. We also notice that the minimum of the negative wave $\varphi$ is a decreasing function of the wave speed, since the derivative of wave height $-\sqrt[3]{10 c /(-a)}$ with respect to wave speed $c$ is less than zero, which means that negative waves $\varphi$ with smaller lowness travel faster.

We claim that the wave profile is symmetric about the vertical axis; that is, we have to prove that $\varphi(\xi)$ is an even function of $\xi$. In order to show this statement, recall (17) and regard $c-a \varphi^{3} / 10$ as a function of $\varphi$. This expression makes sure that for each height of the profile $\varphi$ there exist two values for the steepness of the wave at that point which only differ by sign. Therefore the wave cannot be steeper on one side of the crest than on the other at the same height below the bed.

We claim the negative solitary wave whose inverted profile $|\varphi|$ decays exponentially at infinity; here the absolute is taken because the solitary wave is negative. This can be explained by performing a Taylor expansion of the right-hand side of (17) in $\varphi$ around zero. It follows that $\varphi_{\xi}^{2}=\varphi^{2}+O\left(\varphi^{3}\right)$ as $|\xi| \rightarrow \infty$ and we have

$$
|\varphi(\xi)|=O(\exp (-|\xi|)) \text {, for }|\xi| \longrightarrow \infty
$$

3.2. Local Well-Posedness of (3). To prove the stability of solitary waves, the well-posedness for (3) is firstly required. For the Cauchy problem of (3) we refer to $[37,38]$.

Lemma 4 (see $[37,38]$ ). Assume that $u_{0} \in H^{s}(s>3 / 2)$. Then (3) has a unique solution $u(t)$ in $C\left([0, T], H^{s}\right) \cap C^{1}([0$, $\left.T], H^{s-1}(R)\right)$ such that $u(0)=u_{0}$ for a certain $T>0$. Moreover, the map $u_{0} \rightarrow u\left(\cdot, u_{0}\right)$ is continuous from $H^{s}$ to $C([0, T]$, $\left.H^{s}\right) \cap C^{1}\left([0, T], H^{s-1}(R)\right)$.

\section{Stability}

The method used to verify orbital stability is attributed to Grillakis et al. [42], and we essentially apply a theorem presented therein. To this aim, we list the following assumptions:

(A1) For every $u_{0} \in H^{s}(R), s>3 / 2$, there exists a solution $u$ of $(3)$ in $[0, T)$ such that $u(0)=u_{0}$, where $u \in$ $C\left([0, T) ; H^{s}(R)\right) \cup C^{1}\left([0, T) ; H^{s-1}(R)\right)$. Furthermore, there exist functionals $E(u)$ and $F(u)$ which are conserved for solutions of (3).

(A2) For every $c \in(0, \infty)$, there exists a traveling wave solution $\varphi \in H^{2}$ of (3), where $\varphi<0$ and $\varphi_{\xi} \not \equiv 0$. The mapping $c \rightarrow \varphi(x-c t)$ is $C^{1}\left((0, \infty) ; H^{2}\right)$. Moreover $c E^{\prime}(\varphi)-F^{\prime}(\varphi)=0$, where $E^{\prime}$ and $F^{\prime}$ are the variational derivatives of $E$ and $F$, respectively. 
(A3) For every $c \in(0, \infty)$, the linearized Hamiltonian operator around $\varphi$ defined by

$$
\begin{aligned}
H_{c}: H^{1}(R) & \longrightarrow H^{-1}(R), \\
H_{c} & =c E^{\prime \prime}(\varphi)-F^{\prime \prime}(\varphi)
\end{aligned}
$$

has exactly one negative simple eigenvalue; its kernel is spanned by $\varphi_{\xi}$ and the rest of its spectrum is positive and bounded away from zero.

Theorem 5 (see [42]). Under the assumptions (A1), (A2), and (A3), a solitary wave solution $\varphi(x-c t)$ of (3) is stable if and only if the scalar function $d(c)=c E(\varphi)-F(\varphi)$ is convex in a neighborhood of $c$.

Firstly we verify that (3) satisfies the assumptions (A1)(A3). The assumption (A1) is guaranteed by Lemmas 1 and 4.

To prove that assumptions (A2) and (A3) hold, we calculate the variational derivative of functionals $E(u)$ and $F(u)$ :

$$
\begin{aligned}
& E^{\prime}(u)=\left(1-\partial_{x}^{2}\right) u, \\
& E^{\prime \prime}(u)=1-\partial_{x}^{2}, \\
& F^{\prime}(u)=\frac{a}{4} u^{4}-\frac{1}{2} u_{x}^{2}-u u_{x x}, \\
& F^{\prime \prime}(u)=a u^{3}-u_{x x}+u_{x} \partial_{x}-u \partial_{x}^{2} .
\end{aligned}
$$

Therefore (12) can be rewritten as

$$
c E^{\prime}(\varphi)-F^{\prime}(\varphi)=0 .
$$

Combining (21) with Lemma 3, assumption (A2) is ensured. The linearized Hamiltonian operator $H_{c}$ is given by direct calculation:

$$
\begin{aligned}
H_{c} & =c E^{\prime \prime}(\varphi)-F^{\prime \prime}(\varphi) \\
& =-\partial_{x}\left((c-\varphi) \partial_{x}\right)-a \varphi^{3}+\varphi_{x x}+c .
\end{aligned}
$$

Thus, the corresponding spectrum equation $H_{c} v=\lambda v$ can be written as Sturm-Liouville problem:

$$
-\left(p v_{x}\right)_{x}+(q-\lambda) v=0
$$

where $p=(c-\varphi)$ and $q=c-a \varphi^{3}+\varphi_{x x}$.

Review that a regular Sturm-Liouville system has an infinite many real eigenvalues $\lambda_{0}<\lambda_{1}<\lambda_{2}<\cdots$ with $\lim _{n \rightarrow \infty} \lambda_{n}=\infty$ (see [43]). The eigenfunction $v_{n}(x)$ corresponding to the eigenvalue $\lambda_{n}$ is uniquely determined apart from the different constant factor and has exactly $n$ zeros. Furthermore, via observation, we know that $H_{c}$ is a self-adjoint, second-order differential operator. Hence its eigenvalues $\lambda$ are real and simple, and its essential spectrum is expressed as $[c, \infty)$ owing to the fact that $\lim _{x \rightarrow \infty} q(x)=c$ (see [44]). It can be directly checked that (12) is equivalent to $H_{c}\left(\varphi_{x}\right)=0$. From the properties of the negative solitary waves, we know that $\varphi_{x}$ has exactly one zero on $R$. The above analysis leads us to the conclusion that there is exactly one negative eigenvalue, and the rest of the spectrum is positive and bounded away from zero, which shows that the assumptions are satisfied.

Secondly, we prove that the scalar function $d(c)$ is convex on a neighborhood of $c$.

For differential $d(c)$ with respect to $c$ it follows that

$$
d^{\prime}(c)=\left(c E^{\prime}(\varphi)-F^{\prime}(\varphi), \varphi_{c}\right)+E(\varphi)=E(\varphi) .
$$

Since $\varphi<0$ and $\varphi_{x}=\varphi_{\xi}>0$ in $[0,+\infty)$, it is easy to obtain from (17) that

$$
\varphi=-\varphi_{x} \sqrt{\frac{c-\varphi}{c-a \varphi^{3} / 10}}, \quad \text { for } x \in(0,+\infty) .
$$

Then we calculate the second derivative of $d(c)$ :

$$
\begin{aligned}
& d^{\prime \prime}(c)=\frac{\mathrm{d}}{\mathrm{d} c} \int_{R} \frac{1}{2}\left(\varphi^{2}+\varphi_{x}^{2}\right) \mathrm{d} x \\
& =\frac{\mathrm{d}}{\mathrm{d} c} \int_{0}^{\infty} \varphi^{2}\left(1+\frac{c-a \varphi^{3} / 10}{c-\varphi}\right) \mathrm{d} x \\
& =-\frac{\mathrm{d}}{\mathrm{d} c} \int_{0}^{\infty} \varphi \varphi_{x} \sqrt{\frac{c-\varphi}{c-a \varphi^{3} / 10}}\left(1+\frac{c-a \varphi^{3} / 10}{c-\varphi}\right) \mathrm{d} x .
\end{aligned}
$$

Letting $\varphi_{x} \mathrm{~d} x=\mathrm{d} y$ and $y=\sqrt[3]{10 c / a} z$ we have

$$
\begin{aligned}
& d^{\prime \prime}(c)=-\frac{\mathrm{d}}{\mathrm{d} c} \\
& \cdot \int_{\sqrt[3]{10 c / a}}^{0} y \sqrt{\frac{c-y}{c-a y^{3} / 10}}\left(1+\frac{c-a y^{3} / 10}{c-y}\right) \mathrm{d} y \\
& =\frac{\mathrm{d}}{\mathrm{d} c} \int_{0}^{1}(\sqrt[3]{10 c / a})^{2} \\
& \cdot z \sqrt{\frac{c-\sqrt[3]{10 c / a} z}{c-c z^{3}}}\left(1+\frac{c-c z^{3}}{c-\sqrt[3]{10 c / a} z}\right) \mathrm{d} z=2 \\
& \cdot \frac{\mathrm{d}}{\mathrm{d} c} \int_{0}^{1} \frac{(\sqrt[3]{10 c / a})^{2} z\left(2 c-\sqrt[3]{10 c / a} z-c z^{3}\right)}{\sqrt{(c-\sqrt[3]{10 c / a} z)\left(c-c z^{3}\right)}} \mathrm{d} z \\
& =2 \int_{0}^{1} \frac{f(z)}{g(z)} \mathrm{d} z
\end{aligned}
$$

where $f(z)=\sqrt[3]{c^{2} / a^{2}} z\left(5 \sqrt[3]{c^{2} / a^{2}} z^{2}-\sqrt[3]{100} c^{2}\left(-2+z^{2}\right)+\right.$ $\left.15 c \sqrt[3]{c / a} z\left(-2+z^{3}\right)\right)$ and $g(z)=3 c(\sqrt{c-\sqrt[3]{10 c / a} z})^{3} \sqrt{c-c z^{3}}$. The last step in the above equation is established since

$$
\begin{aligned}
& \frac{\mathrm{d}}{\mathrm{d} c}\left(\frac{(\sqrt[3]{10 c / a})^{2} z\left(2 c-\sqrt[3]{10 c / a} z-c z^{3}\right)}{\sqrt{(c-\sqrt[3]{10 c / a} z)\left(c-c z^{3}\right)}}\right)=\frac{f(z)}{g(z)} \\
& \quad \leq K \frac{1}{\sqrt{1-z}} \in \mathfrak{L}^{1}(0,1),
\end{aligned}
$$

where $K$ is a constant involving parameters $a$ and $c$. 
Since $c>0, a<0$, and $z \in(0,1)$, it can be certified that $f(z)>0, g(z)>0$. Therefore, $d^{\prime \prime}(c)>0$; we come to the conclusion that the negative solitary waves are orbitally stable.

\section{Conflict of Interests}

The authors declare that there is no conflict of interests regarding the publication of this paper.

\section{Acknowledgments}

This work is supported by the National Natural Science Foundation of China (Grant no. 11401096) and Guangdong Province (Grant no. GDJG20141204).

\section{References}

[1] A. S. Fokas and B. Fuchssteiner, "Symplectic structures, their Bäcklund transformations and hereditary symmetries," Physica D: Nonlinear Phenomena, vol. 4, no. 1, pp. 47-66, 1981.

[2] R. Camassa and D. D. Holm, "An integrable shallow water equation with peaked solitons," Physical Review Letters, vol. 71, no. 11, pp. 1661-1664, 1993.

[3] A. Constantin and J. Escher, "Wave breaking for nonlinear nonlocal shallow water equations," Acta Mathematica, vol. 181, no. 2, pp. 229-243, 1998.

[4] C. E. Kenig, G. Ponce, and L. Vega, "Well-posedness and scattering results for the generalized Korteweg-de Vries equation via the contraction principle," Communications on Pure and Applied Mathematics, vol. 46, no. 4, pp. 527-620, 1993.

[5] G. B. Whitham, Linear and Nonlinear Waves, John Wiley \& Sons, New York, NY, USA, 1980.

[6] G. Rodríguez-Blanco, "On the Cauchy problem for the Camassa-Holm equation," Nonlinear Analysis: Theory, Methods \& Applications, vol. 46, no. 3, pp. 309-327, 2001.

[7] A. Constantin, "On the Cauchy problem for the periodic Camassa-Holm equation," Journal of Differential Equations, vol. 141, no. 2, pp. 218-235, 1997.

[8] G. Misiolek, "Classical solutions of the periodic Camassa-Holm equation," Geometric and Functional Analysis, vol. 12, no. 5, pp. 1080-1104, 2002.

[9] Z. Xin and P. Zhang, "On the weak solutions to a shallow water equation," Communications on Pure and Applied Mathematics, vol. 53, no. 11, pp. 1411-1433, 2000.

[10] A. Bressan and A. Constantin, "Global conservative solutions of the Camassa-Holm equation," Archive for Rational Mechanics and Analysis, vol. 183, no. 2, pp. 215-239, 2007.

[11] A. Bressan and A. Constantin, "Global dissipative solutions of the Camassa-Holm equation," Analysis and Applications, vol. 5, no. 1, pp. 1-27, 2007.

[12] H. Holden and X. Raynaud, "Global conservative solutions of the Camassa-Holm equation-a Lagrangian point of view," Communications in Partial Differential Equations, vol. 32, no. 10-12, pp. 1511-1549, 2007.

[13] H. Holden and X. Raynaud, "Dissipative solutions for the Camassa-Holm equation," Discrete and Continuous Dynamical Systems, vol. 24, no. 4, pp. 1047-1112, 2009.

[14] A. Constantin and W. A. Strauss, "Stability of peakons," Communications on Pure and Applied Mathematics, vol. 53, no. 5, pp. 603-610, 2000.
[15] A. Constantin and W. A. Strauss, "Stability of the CamassaHolm solitons," Journal of Nonlinear Science, vol. 12, no. 4, pp. 415-422, 2002.

[16] Z.-Y. Ouyang, S. Zheng, and Z.-R. Liu, "Orbital stability of peakons with nonvanishing boundary for $\mathrm{CH}$ and $\mathrm{CH}-\gamma$ equations," Physics Letters A, vol. 372, no. 47, pp. 7046-7050, 2008.

[17] Y. A. Li and P. J. Olver, "Well-posedness and blow-up solutions for an integrable nonlinearly dispersive model wave equation," Journal of Differential Equations, vol. 162, no. 1, pp. 27-63, 2000.

[18] A. Constantin, "Existence of permanent and breaking waves for a shallow water equation: a geometric approach," Annales de l'Institut Fourier, vol. 50, no. 2, pp. 321-362, 2000.

[19] A. Constantin and J. Escher, "Global existence and blow-up for a shallow water equation," Annali della Scuola Normale Superiore di Pisa-Classe di Scienze, vol. 26, no. 2, pp. 303-328, 1998.

[20] A. Constantin and J. Escher, "Well-posedness, global existence, and blowup phenomena for a periodic quasi-linear hyperbolic equation," Communications on Pure and Applied Mathematics, vol. 51, no. 5, pp. 475-504, 1998.

[21] H. P. McKean, "Breakdown of a shallow water equation," The Asian Journal of Mathematics, vol. 2, no. 4, pp. 867-874, 1998.

[22] J. Yin, L. Tian, and X. Fan, "Stability of negative solitary waves for an integrable modified Camassa-Holm equation," Journal of Mathematical Physics, vol. 51, Article ID 053515, 2010.

[23] W. G. Rui, B. He, S. L. Xie, and Y. Long, "Application of the integral bifurcation method for solving modified CamassaHolm and Degasperis-Procesi equations," Nonlinear Analysis: Theory, Methods \& Applications, vol. 71, no. 7-8, pp. 3459-3470, 2009.

[24] Y. Fu, G. L. Gui, Y. Liu, and C. Z. Qu, "On the Cauchy problem for the integrable modified Camassa-Holm equation with cubic nonlinearity," Journal of Differential Equations, vol. 255, no. 7, pp. 1905-1938, 2013.

[25] Y. Liu, X. Zhu, and J. He, "Factorization technique and new exact solutions for the modified Camassa-Holm and DegasperisProcesi equations," Applied Mathematics and Computation, vol. 217, no. 4, pp. 1658-1665, 2010.

[26] A.-M. Wazwaz, "Solitary wave solutions for modified forms of Degasperis-Procesi and Camassa-Holm equations," Physics Letters A, vol. 352, no. 6, pp. 500-504, 2006.

[27] X. Liu, Y. Liu, and C. Qu, "Orbital stability of the train of peakons for an integrable modified Camassa-Holm equation," Advances in Mathematics, vol. 255, pp. 1-37, 2014.

[28] L. X. Tian and J. L. Yin, "New compacton solutions and solitary wave solutions of fully nonlinear generalized Camassa-Holm equations," Chaos, Solitons and Fractals, vol. 20, no. 2, pp. 289299, 2004.

[29] A.-M. Wazwaz, "A class of nonlinear fourth order variant of a generalized Camassa-Holm equation with compact and noncompact solutions," Applied Mathematics and Computation, vol. 165, no. 2, pp. 485-501, 2005.

[30] A.-M. Wazwaz, "New compact and noncompact solutions for two variants of a modified Camassa-Holm equation," Applied Mathematics and Computation, vol. 163, no. 3, pp. 1165-1179, 2005.

[31] R. A. Kraenkel and A. Zenchuk, "Two-dimensional integrable generalization of the Camassa-Holm equation," Physics Letters A, vol. 260, no. 3-4, pp. 218-224, 1999.

[32] Z. Liu and T. Qian, "Peakons and their bifurcation in a generalized Camassa-Holm equation," International Journal of Bifurcation and Chaos, vol. 11, no. 3, article 781, 2001. 
[33] T. F. Qian and M. Y. Tang, "Peakons and periodic cusp waves in a generalized Camassa-Holm equation," Chaos, Solitons and Fractals, vol. 12, no. 7, pp. 1347-1360, 2001.

[34] Z. Y. Liu and T. F. Qian, "Peakons of the Camassa-Holm equation," Applied Mathematical Modelling, vol. 26, no. 3, pp. 473480, 2002.

[35] L. Tian and X. Song, "New peaked solitary wave solutions of the generalized Camassa-Holm equation," Chaos, Solitons and Fractals, vol. 19, no. 3, pp. 621-637, 2004.

[36] S. A. Khuri, "New ansäz for obtaining wave solutions of the generalized Camassa-Holm equation," Chaos, Solitons and Fractals, vol. 25, no. 3, pp. 705-710, 2005.

[37] Z. Y. Yin, "On the Cauchy problem for the generalized CamassaHolm equation," Nonlinear Analysis: Theory, Methods \& Applications, vol. 66, no. 2, pp. 460-471, 2007.

[38] O. G. Mustafa, "On the Cauchy problem for a generalized Camassa-Holm equation," Nonlinear Analysis. Theory, Methods \& Applications, vol. 64, no. 6, pp. 1382-1399, 2006.

[39] J. Yin, Q. Xing, and L. Tian, "Orbital stability and dynamical behaviors of solitary waves for the Camassa-Holm equation with quartic nonlinearity," Chaos, Solitons \& Fractals, vol. 76, pp. 40-46, 2015.

[40] T. B. Benjamin, “The stability of solitary waves," Proceedings of the Royal Society London Series A: Mathematical, Physical and Engineering Sciences, vol. 328, pp. 153-183, 1972.

[41] T. B. Benjamin, J. L. Bona, and J. J. Mahony, "Model equations for long waves in nonlinear dispersive systems," Philosophical Transactions of the Royal Society of London A, vol. 272, no. 1220, pp. 47-78, 1972.

[42] M. Grillakis, J. Shatah, and W. Strauss, "Stability theory of solitary waves in the presence of symmetry I," Journal of Functional Analysis, vol. 74, no. 1, pp. 160-197, 1987.

[43] G. Birkhoff and G.-C. Rota, Ordinary Differential Equations, John Wiley \& Sons, 1998.

[44] N. Dunford and J. T. Schwarz, Linear Operators, Part II: Spectral Theory, Interscience Publishers, John Wiley and Sons, New York, NY, USA, 1963. 


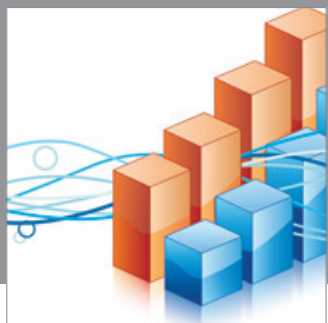

Advances in

Operations Research

mansans

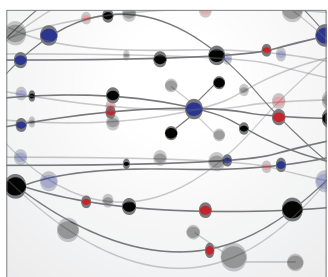

The Scientific World Journal
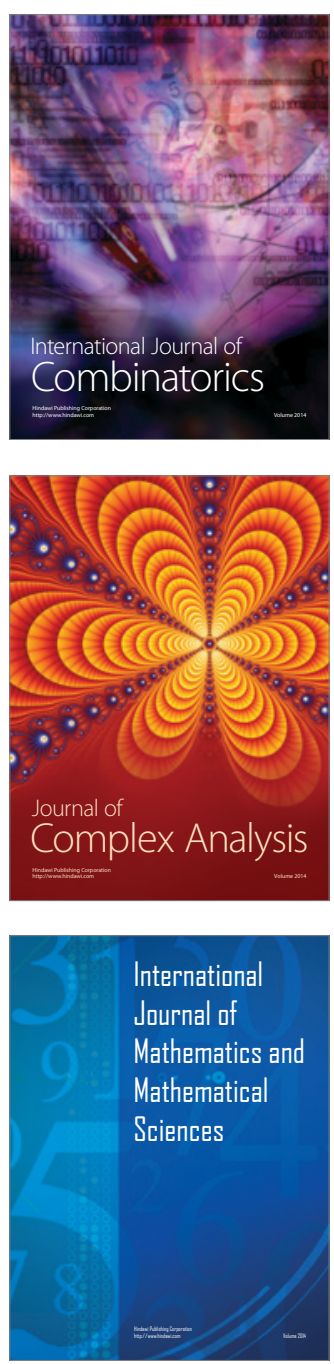
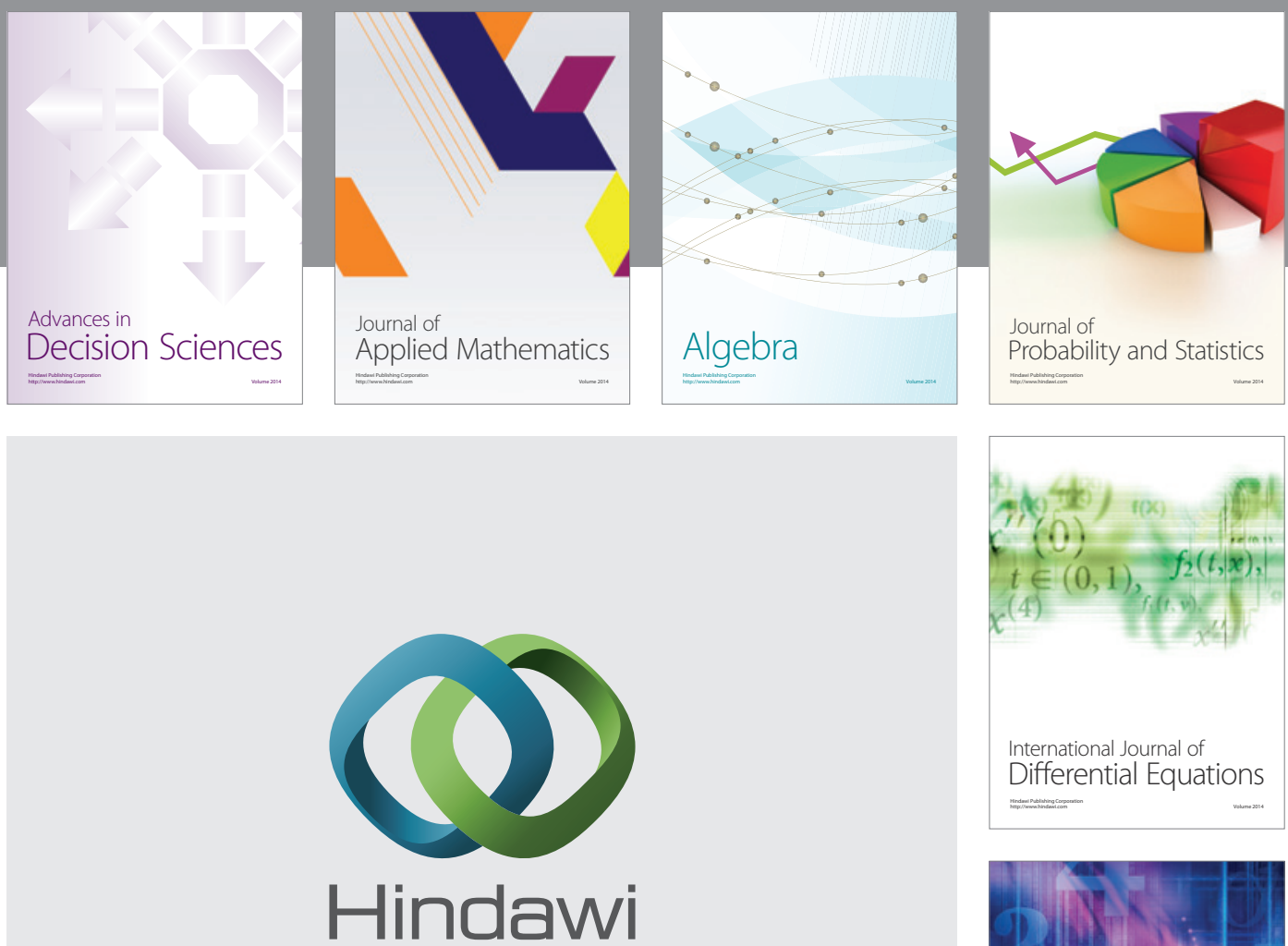

Submit your manuscripts at http://www.hindawi.com
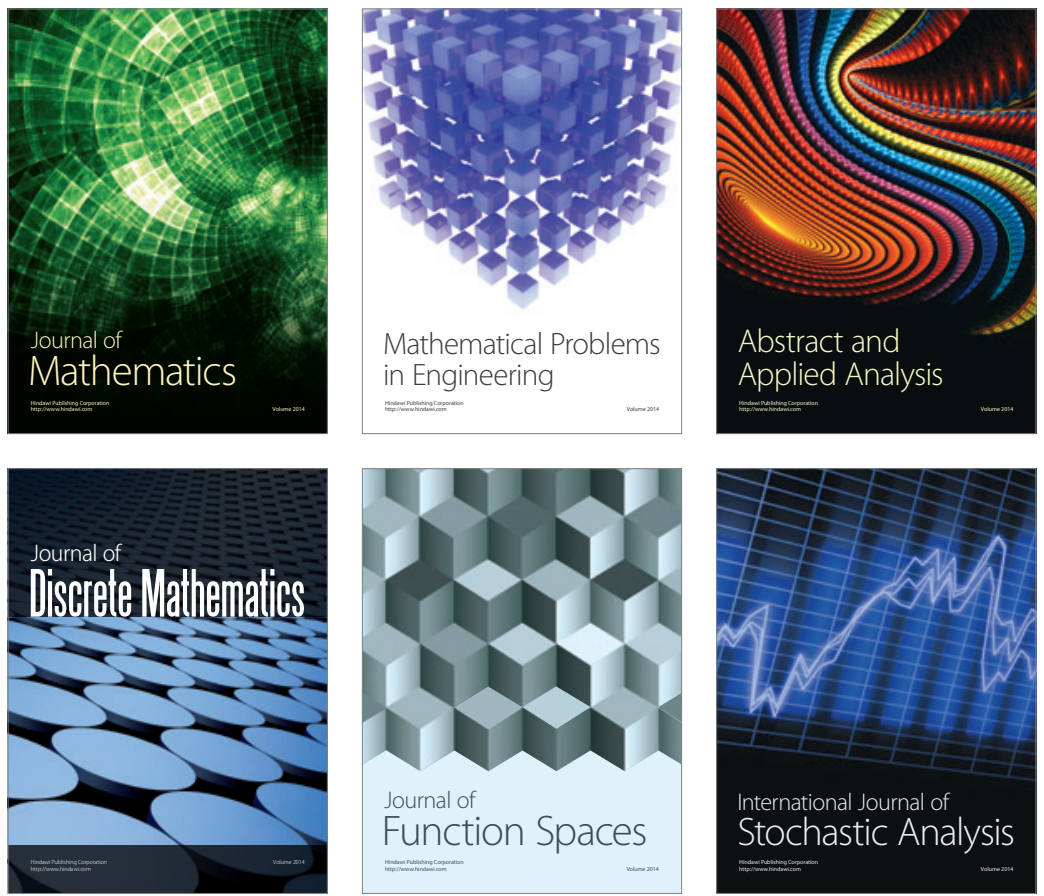

Journal of

Function Spaces

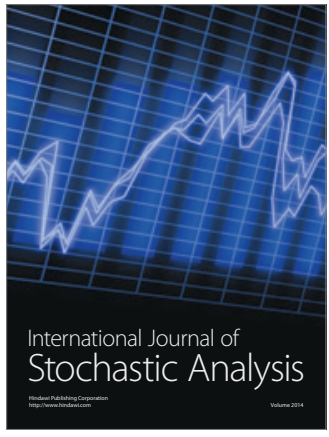

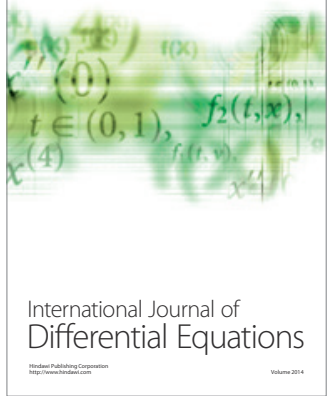
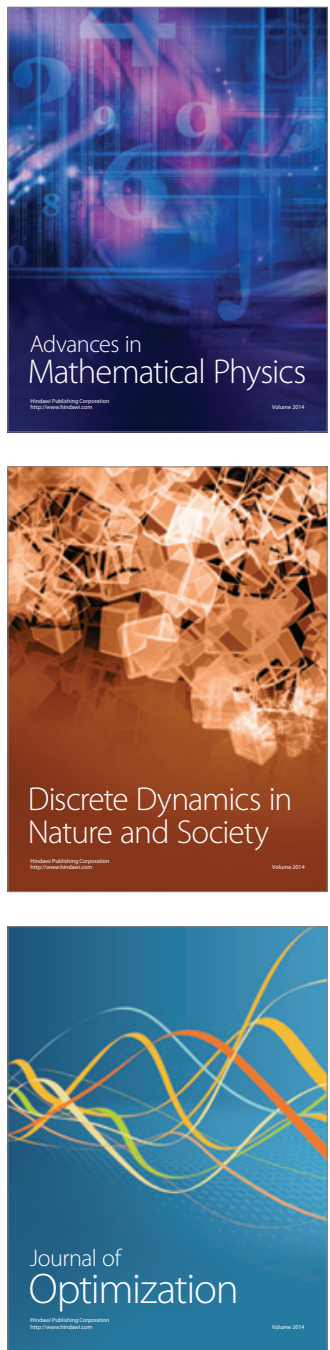